\title{
Effect of three-body interactions on the phase behavior of charge-stabilized colloidal suspensions
}

\author{
A.-P. Hynninen and M. Dijkstra \\ Debye Institute, Soft Condensed Matter, Utrecht University, Princetonplein 5, 3584 CC Utrecht, The Netherlands \\ R. van Roij \\ Institute for Theoretical Physics, Utrecht University, Leuvenlaan 4, 3584 CE Utrecht, The Netherlands
}

(Received 29 January 2004; published 23 June 2004)

\begin{abstract}
We study numerically the effect of attractive triplet interactions on the phase behavior of suspensions of highly charged colloidal particles at low salinity. In our computer simulations, we employ the pair and triplet potentials that were obtained from a numerical Poisson-Boltzmann study [C. Russ et al., Phys. Rev. E 66, 011402 (2002)]. On the basis of free energy calculations, we determine the phase diagram of an aqueous suspension of identical spheres of diameter $\sigma=32 \mathrm{~nm}$ and charge $Z=80$ as a function of colloid concentration and salinity, both for the purely pairwise additive system and for the system with pair and triplet interactions. The main effect of including the triplet interactions is a destabilization of the body-centered-cubic (bcc) crystal phase in favor of the face-centered-cubic (fcc) crystal phase. As a consequence the phase diagram features the coexistence of a rather dilute fluid with an almost-close-packed fcc phase at low salinity and bcc-fcc coexistence with a big density jump at intermediate salinity. The triplet attractions do not affect the phase behavior at sufficiently high salinity; under these conditions the system is well described by the pairwise potential.
\end{abstract}

DOI: 10.1103/PhysRevE.69.061407

PACS number(s): 82.70.Dd, 64.60.Cn, 64.70.-p

\section{INTRODUCTION}

Charge-stabilized colloidal suspensions consist of spherical or anisotropic mesoscopic colloidal particles suspended in a polar solvent with co-ions and counter-ions. A statistical mechanics description of these highly asymmetric multicomponent fluids represents a major challenge as very different length and time scales are involved for the various species. This is the reason why attempts to treat the mesoscopic colloids and the microscopic salt and solvent particles on an equal footing usually fail [1]. It is therefore not surprising that the present understanding of these systems is based on simplified models, in which the degrees of freedom of the microscopic particles have been integrated out, such that the mesoscopic particles interact with an effective (usually pairwise) potential, resulting in a coarse-grained effective onecomponent description of the suspension. The standard and very successful effective one-component description of charged colloidal suspensions dates back to the 1940s and is due to Derjaguin, Landau, Verwey, and Overbeek (DLVO) [2]. The DLVO theory predicts that the effective pair interaction between the colloids consists of a hard-core repulsion due to the finite size of the colloids, screened-Coulomb (Yukawa) repulsion with the screening length given by the Debye length $\kappa^{-1}$ of the electrolyte, and van der Waals attractions with a typical range of a few $\mathrm{nm}$. The screening length $\kappa^{-1}$ defines the thickness of the double layer of opposite charge surrounding each colloidal surface. The range $\kappa^{-1}$ of the screened-Coulomb repulsion is a function of the salt concentration of the electrolyte, the dielectric constant of the solvent, and the temperature. Here we focus on the effect of the salt concentration as this can be tuned over several decades.

At high salt concentrations $\left(\kappa^{-1}\right.$ small $)$ screening of the Coulomb interactions is most efficient and the van der Waals attractions become important, which explain reversible vapor-liquid phase separation or irreversible flocculation as observed experimentally $[3,4]$. In the low-salt regime $\left(\kappa^{-1}\right.$ large) van der Waals attractions are completely masked by the much longer-ranged screened Coulomb repulsions. The repulsive long-range nature of the interactions in the low-salt regime explains the observation of stable crystal phases at packing fractions of a few percent [5]. The success of the DLVO theory on explaining experimental observations made it a true cornerstone of colloid science.

Within the past decade, some experimental observations have been published that question the validity of the DLVO theory. Without exception these experiments were done in the low-salt regime and they include observations of a broad gas-solid coexistence [6], "voids" of vapor in otherwise homogeneous suspension $[7,8]$, long-lived dense metastable crystallites [8,9], and even a gas-liquid coexistence [10] (although not without some dispute [11]).

A long-range attraction between like-charged spheres would account naturally for these experimental observations, but would be in contradiction with the long-accepted DLVO theory. This discrepancy triggered intense theoretical and experimental activities and vivid debates. For more details on the current state of affairs, the reader is referred to the review papers in [12].

While many of these experiments have proven to be difficult to reproduce, there are, let us say, more "traditional" experiments on charged colloids, which until now were considered to include no information that contradicts the DLVO theory but which on a closer examination do. These experiments include the ones made by Monovoukas and Gast [13], Sirota et al. [14], and Schöpe et al. [15]. What all three of them have in common is the observation of a broad gas-solid or solid-solid coexistence in the low-salt regime. Monovoukas and Gast observed a gas-solid coexistence with a density jump of around 200\%, while Sirota et al. and Schöpe et al. 
reported a bcc-fcc coexistence with density jumps of $26 \%$ and $72 \%$, respectively. To appreciate the broadness of these coexistence regions, one recalls that the fluid-fcc density jump of the hard spheres is only $10 \%$. This can be considered as the maximum density jump allowed by the DLVO theory and can only take place at a very high salt concentration. In the low-salt regime, the DLVO theory predicts soft long-ranged repulsive interactions and it is well known that such interactions give rise to extremely narrow coexisting regions [16-18]. The observations of broad coexisting regions therefore call for a long-ranged attraction.

Several mechanisms have been proposed for the source of attraction in charge-stabilized colloidal suspensions. They can be divided into two classes: those trying to improve the DLVO theory (which is a mean-field theory) by including correlations between the micro-ions and those where (colloidal) many-body effects are considered. The focus here is on the latter.

Indeed, at low salt concentration, of the order of several $\mu \mathrm{M}$ 's, the double-layer thickness is of the order of the colloid diameter-i.e., $\kappa^{-1} \geqslant \sigma$-and pairwise additivity might break down as many-body interactions become important. In some of the theoretical approaches the many-body contributions are captured in so-called volume terms, which do not depend on the particle coordinates but on the density of the system [19-23]. Alternatively, one captures the many-body effect by many-body potentials-i.e., functions of the colloidal coordinates but not of colloid density.

Recent experimental $[24,25]$ and theoretical $[26,27]$ results support the existence of many-body effects. Both studies show that the pair repulsion is reduced at distances larger than the mean distance between the colloids, due to manybody interactions. The reduction of the repulsion at distances larger than the mean distance between the colloids can be traced back to the shielding effect of the macro-ions. This is in line with recent explicit numerical calculations within nonlinear Poisson-Boltzmann theory, which show that the pairwise repulsion between two colloids is dramatically reduced when a third colloid is placed in between [26]. This effect results in effective attractive triplet interactions between the charged colloidal spheres. At high salt concentrations this effect is not important, since the double layers are small and the Yukawa repulsion has already decayed at the distance between the outermost colloids (two diameters or more). However, at low salt concentrations the double layers are large and a considerable reduction of the repulsion between the outermost colloids is caused by the presence of the third intermediate colloid: pairwise additivity breaks down and many-body interactions become important. Based on numerical calculations, simple empirical expressions were obtained for the pair and triplet interactions within the nonlinear Poisson-Boltzmann theory, specifically in the nonlinear regime of low salt concentrations and high charges [26]. Moreover, it was shown within a van der Waals-like meanfield theory that the strength of the attractive three-body interaction is large enough to induce gas-liquid phase coexistence.

In this paper, we use computer simulations to study the effect of attractive three-body interactions on the phase behavior of charge-stabilized colloidal suspensions. We deter- mine the phase diagram using free energy calculations both for an effective one-component system of colloids interacting with pair and triplet interactions and that of a pairwise system without the three-body interactions. We find that attractive three-body interactions drive most likely a gas-solid transition instead of a gas-liquid transition as was found previously within the van der Waals-like mean-field theory [26]. Moreover, the presence of triplet attractions also affects the relative stability of fcc and bcc crystals. A brief version of this paper was published elsewhere [28].

The paper is organized as follows. In Sec. II we describe our system. In Sec. III, we briefly describe the simulation techniques used. We present the phase diagrams in Sec. IV, and we make some concluding remarks in Sec. V.

\section{MODEL}

Our model for the colloidal suspension consists of $N$ identical spherical colloidal particles with a hard-core diameter $\sigma$ and a uniformly distributed surface charge of $-Z e$, immersed in a volume $V$, together with $N Z$ monovalent counter-ions, and added salt at a bulk (reservoir) concentration of $c_{s}$. The unit (proton) charge is denoted by $e$. The monovalent positive counter-ions and negative co-ions are assumed to be charged-point particles. The electrolyte is treated as a structureless continuum characterized by the dielectric constant $\epsilon$. Direct simulation of such a system that treats all species on an equal footing is untractable for large $Z$, due to the different length and time scales associated with the mesoscopic and microscopic species. This problem may be circumvented by adopting a coarse-grained description for the micro-ions. In this approach, the micro-ions are not treated individually, but at the level of their equilibrium density distributions $\rho_{ \pm}(\mathbf{r})$ for the positive and negative micro-ions in a fixed configuration of the colloids. Due to large differences in time scales, the micro-ions may be assumed to be in thermodynamic equilibrium for any configuration of the colloids. The inhomogeneous density distributions $\rho_{ \pm}(\mathbf{r})$ in the presence of a fixed configuration of colloidal particles can be obtained by solving the nonlinear Poisson-Boltzmann equation. It is convenient to treat the electrolyte grand canonically-i.e.. at a fixed chemical potential of salt, $\mu_{s}$. Once $\rho_{ \pm}(\mathbf{r})$ have been determined, the effective interactions between the colloids are given by

$$
V_{\text {eff }}\left(\left\{\mathbf{r}_{i}\right\}\right)=\sum_{i<j}^{N} v\left(\mathbf{r}_{i}, \mathbf{r}_{j}\right)+\Omega\left(\{\mathbf{r}\}, N, \mu_{s}\right),
$$

where $v\left(\mathbf{r}_{i}, \mathbf{r}_{j}\right)$ is the nonelectrostatic part of the pair potential between colloids $i$ and $j$, the center-of-mass coordinates of the colloids are given by $\mathbf{r}_{i}$ with $i=1, \ldots, N$, and $\Omega_{N}$ $=\Omega\left(\left\{\mathbf{r}_{i}\right\}, N, \mu_{s}\right)$ is the grand potential of the electrolyte in the external field of a fixed configuration of $N$ colloidal particles. In the present case the nonelectrostatic part $v\left(\mathbf{r}_{i}, \mathbf{r}_{j}\right)$ is equal to the hard-core interaction-i.e., $v\left(\mathbf{r}_{i}, \mathbf{r}_{j}\right)=v\left(r_{i j}\right)=\infty$, if $r_{i j}$ $=\left|\mathbf{r}_{i}-\mathbf{r}_{j}\right|<\sigma$, and $v\left(r_{i j}\right)=0$ otherwise. In principle, the effective interactions can be calculated "on the fly" for each colloid configuration in the same spirit as the ab initio method of Car and Parrinello for ion-electron systems [29] (applica- 
tions for colloidal systems are found in Refs. [30,31]). However, here we adopt a different method based on effective $n$-body interactions. It can be shown explicitly that $\Omega_{N}$ can be uniquely decomposed into effective $n$-body interactions $\Omega^{(n)}$ between the colloids [26,32]:

$$
\Omega_{N}=N \Omega^{(1)}+\sum_{i<j}^{N} \Omega^{(2)}\left(\mathbf{r}_{i}, \mathbf{r}_{j}\right)+\sum_{i<j<k}^{N} \Omega^{(3)}\left(\mathbf{r}_{i}, \mathbf{r}_{j}, \mathbf{r}_{k}\right)+\cdots,
$$

where the ellipsis represents the terms $\Omega^{(n)}$ for $n \geqslant 4$. The $n$-body potentials are defined in a system with $n$ colloids with neutralizing counterions in contact with a salt reservoir at chemical potential $\mu_{s}$. The one-body potential $\Omega^{(1)}$ is actually the grand-potential difference between a sea of salt at $\mu_{s}$, volume $V$ with and without a single colloid; it can be interpreted as the self-energy of a colloid or the Henry coefficient. The effective pair potential $\Omega^{(2)}\left(\mathbf{r}_{i}, \mathbf{r}_{j}\right)$ between two colloids is the grand-potential difference between a system with volume $V$ containing two colloids at separation $r_{i j}=\mid \mathbf{r}_{i}$ $-\mathbf{r}_{j} \mid$ and at infinite separation. For $n>2$, the effective $n$-body interactions can be defined along the same lines and will depend on the exact coordinates of $n$ colloids. It is worth noting that the mapping of $\Omega_{N}$ onto effective $n$-body interactions with $n=1, \ldots, N$ is exact. One actually hopes, of course, that the rate of convergence of the expansion is fast and that $\Omega_{N}$ can be approximated by only including $n$-body potentials of $n=1,2, \ldots, n^{*} \ll N$. In many cases, the expansion is truncated at pair potential level, and three- and higher-body interactions are ignored. Recently, two- and three-body interactions were calculated numerically within Poisson-Boltzmann theory [26]. The effective pair interactions turn out to be purely repulsive and can be fitted by a screened Coulomb potential, consistent with the well-known DLVO result:

$$
\beta \Omega^{(2)}(r)=A^{(2)} \frac{\lambda_{B}}{\sigma} \frac{\exp [-\kappa r]}{r / \sigma} .
$$

Here $A^{(2)}$ is a fitting constant, $\kappa=\sqrt{8 \pi \lambda_{B} c_{S}}$ is the inverse Debye screening length, $\lambda_{B}=e^{2} \beta / \epsilon$ is the Bjerrum length, and $\beta=1 / k T$. By contrast, it was found in Ref. [26] that the three-body interactions are purely attractive in all considered cases. More surprisingly, the triplet interaction calculated for many configurations of the three colloids collapsed onto one master curve that can be fitted remarkably well by a Yukawa potential which depends on the sum of the three distances, $L=r_{i j}+r_{j k}+r_{i k}$, instead of the three distances separately:

$$
\beta \Omega^{(3)}(L)=-A^{(3)} \frac{\lambda_{B}}{\sigma} \frac{\exp [-\gamma L]}{L / \sigma},
$$

where the two fit parameters are the decay constant $\gamma$ and the prefactor $A^{(3)}$. The prefactors $A^{(2)}$ and $A^{(3)}$ as well as the decay length $\gamma$ are given in Ref. [26] for different combinations of the reduced charge $\bar{Z}=Z \lambda_{B} / \sigma$ and $\kappa \sigma$. These empirical fits for the two- and three-body potentials enable us to perform computer simulations and study the effect of attrac- tive three-body potentials as the lowest-order correction to pairwise additivity.

\section{METHODS}

Our purpose is to calculate the Helmholtz free energy of a system with a Hamiltonian that includes a potential energy that is given as a sum of the pair and triplet potential energies of Eqs. (3) and (4), respectively, and of the nonelectrostatic hard-core contribution $v\left(r_{i j}\right)$. That is, the potential energy function $U_{23}\left(\mathbf{r}^{N}\right)$ of our Hamiltonian is written as

$$
U_{23}\left(\mathbf{r}^{N}\right)=\sum_{i<j}^{N}\left[v\left(r_{i j}\right)+\Omega^{(2)}\left(r_{i j}\right)\right]+\sum_{i<j<k}^{N} \Omega^{(3)}\left(r_{i j}+r_{j k}+r_{i k}\right) .
$$

Note that the kinetic energy term in the Hamiltonian plays no role in phase stability and can therefore be ignored. A cubic simulation box with periodic boundary conditions is used and the minimum image rule is applied. While the minimum image rule works fine for pair potentials, there is no unique way of applying the minimum-image convention to a group of three particles [33]. Consider a triangle of particles labeled by $i, j$, and $k$. The minimum-image convention can only be applied to two sides of the triangle — say, to particle $j$ with $i$ and to particle $k$ with $i$. The distance between particle $j$ and $k$ is then fixed. The distance between the closest image of particles $j$ and $k$ with respect to particle $i$ is not necessarily the minimum-image distance of $j$ to $k$. This can easily be shown by defining the $x$ component of the translation vector,

$$
t_{i j}=\left[\left(x_{i}-x_{j}\right) / L\right] L, \quad t_{i k}=\left[\left(x_{i}-x_{k}\right) / L\right] L,
$$

with $L$ the box length and $[x]$ the closest integer to $x$. The resulting $x$ components of the separation vector between the particles are given by

$$
\begin{gathered}
x_{i j}=x_{i}-x_{j}-t_{i j}, \\
x_{i k}=x_{i}-x_{k}-t_{i k}, \\
x_{j k}=x_{j}-x_{k}+t_{i j}-t_{i k},
\end{gathered}
$$

and similarly for the $y$ and $z$ components. For most cases $x_{j k}$ is the same as given by the minimum-image conventioni.e., $x_{j k}=x_{j}-x_{k}-t_{j k}$-but for those cases where this is not true one should check if the total side length of the triangle can be minimized by applying the minimum-image convention to the other sides of the triangle. In fact there are at most three possible choices of triangles that have at least two sides with lengths less than half the box length, as the minimum-image convention can only be applied to two sides of the triangle. However, it is too time-consuming to check all possible triangles in each Monte Carlo step, and we have therefore chosen for a simpler minimum-image rule. Our minimum-image rule for the triplets is best explained and motivated by considering the way the potential energy is calculated: We first take particle $i$ ( $N$ choices); we then take particle $j$ (from the set of $N-1$ particles that are left) and apply the minimumimage rule for particles $i$ and $j$. Next the two-body potential 
$\Omega^{(2)}$ is calculated for the pair $i j$. For each pair $i j$ we take a third particle $k$ (from the set of $N-2$ particles that are left). Since now the distance between $i$ and $j$ is already fixed, we only have two choices for the minimum image of $k$; i.e., we can take the minimum image of $k$ with respect to $i$ or with respect to $j$. By choosing the minimum image of $k$ that gives the triangle with the smallest total side length of the triangle, we take into account the position of particle $k$ that screens the pair interaction between particles $i$ and $j$ most.

A consequence of this three-body minimum-image rule is that the resulting three-body potential energy $\sum_{i<j<k}^{N} \Omega^{(3)}$ $\times\left(r_{i j}+r_{j k}+r_{i k}\right)$ depends on the order in which the summation is done. This is why the result is not (in all geometries) equal to one-sixth of the unrestricted sum $\Sigma_{i, j, k}^{N} \Omega^{(3)}\left(r_{i j}+r_{j k}+r_{i k}\right)$, where all triangles are calculated 6 times. However, according to our tests, for a system with an adequate size the error produced is at least five orders of magnitude smaller than the error produced by statistical noise.

The two-body potential is truncated at a cutoff radius $r_{c}$, which is chosen to be equal to the half of the box length. For the three-body potential we choose to truncate the potential when one of the side lengths of the minimum-image triangle is greater than $r_{c}$ (the same method as used in Ref. [33]).

As the free energy cannot be measured directly in a Monte Carlo (MC) simulation, we used thermodynamic integration to relate the free energy of the system interacting with a potential energy function $U_{23}\left(\mathbf{r}^{N}\right)$ given by Eq. (5) to that of a reference system at the same density. The Helmholtz free energy of the solid phases is calculated using the Frenkel-Ladd method $[34,35]$. To this end we introduce the auxiliary potential energy function

$$
\beta U_{\mathrm{sol}}^{\lambda}\left(\mathbf{r}^{N}\right)=\beta U_{23}\left(\mathbf{r}^{N}\right)+\lambda \sum_{i=1}^{N}\left(\mathbf{r}_{i}-\mathbf{r}_{0, i}\right)^{2} / \sigma^{2}
$$

where $\mathbf{r}_{0, i}$ is the lattice position of particle $i$, and $\lambda$ the dimensionless switching parameter. In Eq. (7), particles are coupled to their lattice sites with harmonic springs: for $\lambda$ $=0$ we recover the system of interest, while for a sufficiently high value of $\lambda$-say, $\lambda=\lambda_{\mathrm{m}}$ - the particles do not "feel" each other and the system reduces to that of a noninteracting Einstein solid with Madelung energy $U_{23}\left(\mathbf{r}_{0}^{N}\right)$-i.e., the potential energy of a crystal with all particles at their lattice positions. It is a standard result that $[34,36]$

$$
\begin{aligned}
\beta F_{\mathrm{sol}}(N, V, T)= & \beta F_{\mathrm{Ein}}^{\mathrm{CM}}\left(N, V, T, \lambda_{\mathrm{m}}\right)+\beta F_{\text {corr }}(N, V, T) \\
& -\int_{0}^{\lambda_{\mathrm{m}}} d \lambda\left\langle\sum_{i=1}^{N}\left(\mathbf{r}_{i}-\mathbf{r}_{0, i}\right)^{2} / \sigma^{2}\right\rangle_{\lambda}^{\mathrm{CM}},
\end{aligned}
$$

where the superscript "CM" on the ensemble average denotes that it is calculated for a crystal with fixed center of mass. The free energy of an Einstein crystal with fixed center of mass is given by

$$
\begin{aligned}
\beta F_{\text {Ein }}^{\mathrm{CM}}\left(N, V, T, \lambda_{\mathrm{m}}\right)= & \beta U_{23}\left(\mathbf{r}_{0}^{N}\right)-\frac{3(N-1)}{2} \ln \left[\frac{\pi}{\lambda_{\mathrm{m}}}\right] \\
& +(N-1) \ln \left[\frac{\Lambda^{3}}{\sigma^{3}}\right],
\end{aligned}
$$

where $\Lambda$ is the de Broglie wavelength. The correction term $F_{\text {corr }}$ arises when the constraint on the center of masses is released-i.e., the Helmholtz free energy difference between the unconstrained and constrained crystal:

$$
\beta F_{\text {corr }}(N, V, T)=\ln \left[\frac{\Lambda^{3}}{V N^{1 / 2}}\right] .
$$

In our calculations we have replaced $U_{23}\left(\mathbf{r}_{0}^{N}\right)$ with $N$ times the Madelung energy per particle obtained from an "infinite" (i.e., large enough) crystal-i.e., $u_{M}\left(N_{\infty}\right) \equiv U_{23}\left(\mathbf{r}_{0}^{N_{\infty}}\right) / N_{\infty}$ with $N_{\infty}(\approx 5000-20000)$ particles. For these system sizes, the tail correction to the potential energy due to the finite cutoff radius can be neglected. Therefore, no further tail corrections to the potential energy are needed. Note, however, that there is still a nonzero finite-size correction to the free energy from two other sources: the implicit effect of the cutoff to the value of $\left\langle r^{2}\right\rangle_{\lambda}^{\mathrm{CM}}$ and the effect of finite number of particles [36].

The Helmholtz free energy of the fluid phase is calculated using the $\lambda$ integration with the hard-sphere fluid as a reference state [34]. To this end, we introduce an auxiliary potential energy function

$$
U_{\text {fluid }}^{\lambda}\left(\mathbf{r}^{N}\right)=\sum_{i<j}^{N} v\left(r_{i j}\right)+\lambda\left[U_{23}\left(\mathbf{r}^{N}\right)-\sum_{i<j}^{N} v\left(r_{i j}\right)\right],
$$

where $0 \leqslant \lambda \leqslant 1$ is the coupling parameter: at $\lambda=0$ the interaction reduces to that of a fluid of $N$ hard spheres, while at $\lambda=1$ it is the potential energy function of interest (for fixed $V)$. The Helmholtz free energy is

$$
\begin{aligned}
F_{\text {fluid }}(N, V, T)= & F_{\text {fluid }}^{\mathrm{HS}}(N, V, T) \\
& +\int_{0}^{1}\left\langle U_{23}\left(\mathbf{r}^{N}\right)-\sum_{i<j}^{N} v\left(r_{i j}\right)\right\rangle_{\lambda} d \lambda,
\end{aligned}
$$

where $F_{\text {fluid }}^{\mathrm{HS}}$ is the free energy of a hard-sphere fluid, for which we use the Carnahan-Starling expression [37]

$$
\frac{\beta F_{\text {fluid }}^{\mathrm{HS}}}{N}=\ln \left[\frac{N \Lambda^{3}}{V}\right]-1+\frac{\eta(4-3 \eta)}{(1-\eta)^{2}} .
$$

In the case of solids, the tail correction for the potential energy could be calculated exactly from the Madelung energy for an infinite system. This is not possible for a fluid, and therefore the fluid free energy calculation inevitably suffers from the finite cutoff radius $r_{c}$ for the potential energy. For a system with only pair interactions this problem can be solved by adding a tail contribution to the potential energy which is approximated by the potential energy due to a continuous distribution of particles beyond the cutoff radius $r_{c}$-i.e., by setting $g(r)=1$ for $r>r_{c}$ [34]. A similar tail correction for the three-body potential $\Omega^{(3)}$ is not very practical 
as it requires a numerical integration over a threedimensional region and the approximation $g(r)=1$ is not accurate because some triangles consist of particle pairs with $r<r_{c}$. Therefore, we approximate the tail correction per particle, $u_{\text {tail }}$, for a fluid with two- and three-body interactions by the difference between the Madelung energy per particle of an "infinite" system $u_{M}\left(N_{\infty}\right)$ and that with the same number of particles as the simulated fluid $u_{M}(N)$-i.e., $u_{\text {tail }}$ $=u_{M}\left(N_{\infty}\right)-u_{M}(N)$.

The integrations in Eqs. (8) and (12) were done using the Gauss-Legendre quadrature with 10 integrations points. This number of integration points was tested to be adequate by recalculating some of the results with more (20 or 32) integration points. The number of particles was chosen to be $N$ $=250$ for the bcc and $N=256$ for the fcc and fluid phases.

To improve the accuracy of the numerical quadrature, we change the integrand for the solid in Eq. (8) in a slowly varying function by changing the integration variable from $\lambda$ to $\ln (c+\lambda)$ as described in Ref. [34]. The value of $c$ $=N \sigma^{2} /\left\langle\sum_{i=1}^{N}\left(\mathbf{r}_{i}-\mathbf{r}_{0, i}\right)^{2}\right\rangle_{\lambda=0}^{\mathrm{CM}}$ was evaluated in the beginning of the free energy calculation and the value of the parameter $\lambda_{\mathrm{m}}$ was chosen to be large enough so that for $\lambda=\lambda_{\mathrm{m}}$ the system behaves as an Einstein crystal.

Each evaluation of the integrands in Eqs. (8) and (12), respectively, consisted of an equilibration run and a sampling run, both with $N_{t}=1000-2000$ trial moves per particle. For systems interacting with a potential energy given by Eq. (5), the evaluation of the three-body interaction scales with $N^{3}$. Our choice of $N_{t}$ resulted in MC simulations that took about $36 \mathrm{CPU}$ hours per free energy point on a supercomputer. In order to map out the phase diagram the Helmholtz free energy must be determined for the fluid, bcc, and fcc phases and for many statepoints-i.e., about 10 different packing fractions and about 8 different values of salt concentrations or $\kappa \sigma$, which resulted in approximately $1 \mathrm{yr}$ of CPU time on a supercomputer. For the two-body system the number of trials was often increased to $N_{t}=10000$ per particle. At small values of $\lambda$ the data become more noisy and therefore, in order to reach better statistical accuracy, the number of trial moves, $N_{t}$, was doubled for the three integration points with smallest value of $\lambda$ and for estimation of the parameter $c$ (the $\lambda=0$ point).

\section{RESULTS}

Using the methods described in Sec. III, we performed free energy calculations for given $\kappa \sigma$ and $\bar{Z}=Z \lambda_{B} / \sigma$ with the parameters $A^{(2)}, A^{(3)}$, and $\gamma$ taken from Ref. [26]. Common tangent construction was used to determine the phase coexistence from the free energy data. We chose the ratio between the Bjerrum length and the colloidal diameter to be $\lambda_{B} / \sigma$ $=0.0225$ and focused on calculating the phase behavior for reduced charge $\bar{Z}=1.8$. This corresponds to charge $Z=80$ and, in water at room temperature (for which $\lambda_{B}=7.2 \AA$ ), to diameter $\sigma=32 \mathrm{~nm}$. Phase diagrams are presented in the $\eta$ $\kappa \sigma$ representation, where $\eta=\pi \sigma^{3} N / 6 V$ is the colloidal packing fraction. Note that $\kappa$ does not depend on $\eta$. We note that our parameters are in the regime, where, according to the

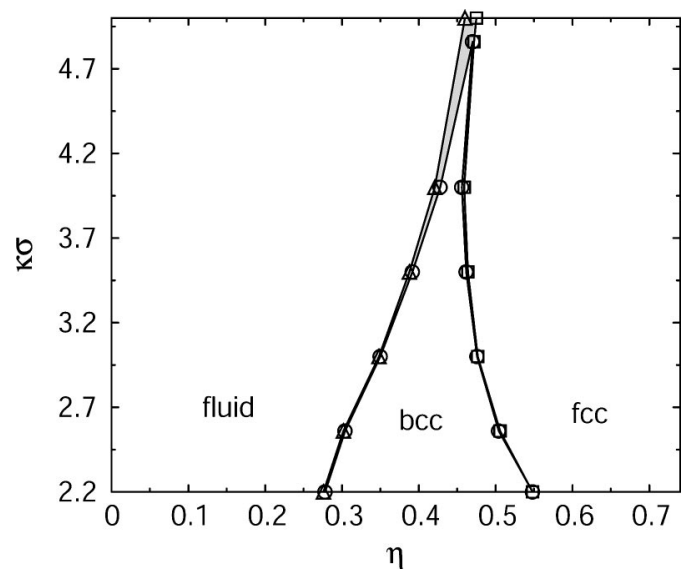

FIG. 1. Phase diagram for $\bar{Z}=1.8$ including only the two-body interactions. The gray color represents a coexistence region, while triangles $(\triangle)$, circles $(\bigcirc)$, and squares $(\square)$ represent the fluid, bcc, and fcc phase boundaries, respectively.

theory in Ref. [38], the effective charge is close to the bare charge: For $\kappa \sigma$ from 2.2 to 2.56 , the theory predicts an effective charge of $Z_{\text {eff }}=75$, which is close to our bare charge of $Z=80$.

In Fig. 1 we present the phase diagram for a system including only the two-body interactions-i.e., for the potential energy of Eq. (5) where the three-body term is disregarded. The coexistence regions are colored gray, and tie lines between the coexisting phases are horizontal. The prefactors $A^{(2)}$ for $\kappa \sigma>2.56$ were obtained by linear extrapolation with respect to $\kappa \sigma$, since the maximum value of $\kappa \sigma$ for which the two-body prefactor $A^{(2)}$ was determined in Ref. [26] is given by $\kappa \sigma=2.56$.

As can be seen from Fig. 1, the bcc phase is very much favored at low values of $\kappa \sigma$ (low salt), while the bcc regime ends in an upper triple point (position of which is given here only as a rough estimate) at $\kappa \sigma \simeq 4.86$. We compared our fluid-bcc coexistence line with the one given for pointYukawa particles in Ref. [39] and found them to be in good agreement in the low- $\kappa \sigma$ regime, where the hard core is not expected to play a role. Note also from Fig. 1 that the fluidbcc and bcc-fcc coexistence regions shrink when moving to the lower values of $\kappa \sigma$, as expected since the potential becomes softer.

In Fig. 2 we present the phase diagram for the same system but now with two- and three-body interactions-i.e., for the full potential in Eq. (5). Note the different $\kappa \sigma$ scale in Figs. 1 and 2. Because the values of the parameters $A^{(2)}, A^{(3)}$, and $\gamma$ are only given in Ref. [26] for $\kappa \sigma=2.0$ and $\kappa \sigma=2.56$, linear interpolation with respect to $\kappa \sigma$ was used to get the parameter values at intermediate $\kappa \sigma$.

Figure 2 shows a very broad fluid-fcc coexistence in the low-salt regime $\kappa \sigma \lesssim 2.36$-i.e., below a fluid-bcc-fcc triple point (the horizontal dashed line in Fig. 2). In the regime $2.36 \lesssim \kappa \sigma \lesssim 2.43$-i.e., above the triple point-there is a very narrow fluid-bcc coexistence at $\eta \approx 0.35$ and a rather broad bcc-fcc coexistence at higher $\eta$. The density jump of the latter shrinks upon approach of $\kappa \sigma \approx 2.43$ and goes to a negligible value for $\kappa \sigma>2.43$. 


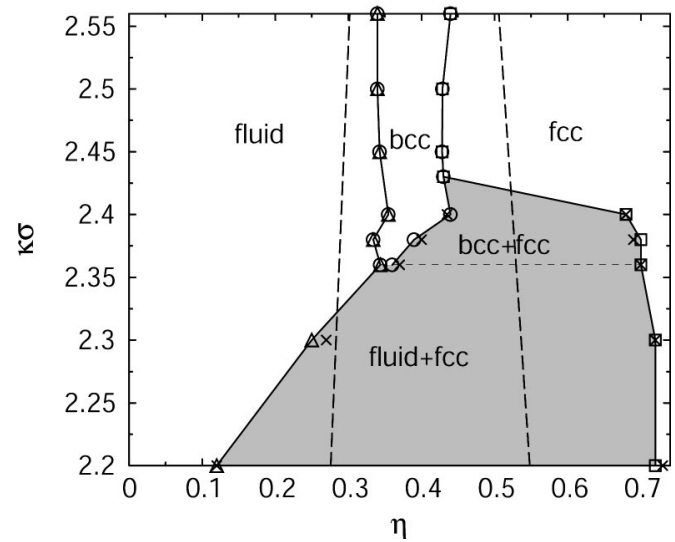

FIG. 2. Phase diagram for $\bar{Z}=1.8$ including the two- and threebody interactions. The gray color represents a coexistence region, while triangles $(\triangle)$, circles $(\bigcirc)$, and squares $(\square)$ represent the fluid, bcc, and fcc phase boundaries, respectively. The crosses $(X)$ mark the bcc-fcc and fluid-fcc coexistence points based on Eq. (14). The two vertical long-dashed lines represent the fluid-bcc and bccfcc phase boundaries of the system with only two-body interactions (see Fig. 1). The horizontal dashed line denotes the fluid-bcc-fcc triple point.

The essentially vertical long-dashed lines in Fig. 2 give the fluid-bcc and bcc-fcc phase boundaries of the system where only two-body interactions are present (Fig. 1). We observe that the inclusion of the three-body interactions reduces the stability of the bcc phase in the regime 2.36 $\lesssim \kappa \sigma \lesssim 2.56$ and closes up the bcc pocket at $\kappa \sigma \approx 2.36$.

In the limit of high salt, the phase diagram of the system with the two- and three-body interactions (Fig. 2) should converge to the phase diagram of the system with only the two-body interactions (Fig. 1). In order to investigate this, we calculated the Madelung energies per particle at $\kappa \sigma$ $=4.0$ for both systems and found them to be very close to each other. Therefore, we expect that at $\kappa \sigma>4.0$ the phase diagram of Fig. 2 reduces to the two-body phase diagram of Fig. 1.

We have checked the effect of the tail corrections used for the fluid phase on the phase behavior in Fig. 2. We find that the tail correction in the fluid phase is unimportant for the broad fluid-fcc phase coexistence. Also, the fluid-bcc phase boundary is hardly affected by the tail correction: If we remove the tail correction from both the fluid and bcc phases, the fluid-bcc phase boundary moves to lower packing fraction by about 0.002 .

In Fig. 3 we show as an example the free energy data for $\kappa \sigma=2.2$, which were used to determine the phase coexistence in the system with two- and three-body interactions (Fig. 2). A common tangent construction reveals a coexistence between a fluid at packing fraction $\eta \approx 0.12$ and an fcc solid at packing fraction $\eta \approx 0.72$. Because the dip in the fcc free energy at $\eta \approx 0.72$ is very deep, the underlying fluid-bcc coexistence is only metastable and the same is true for the possible gas-liquid coexistence.

In order to study the source of this dip we made a very simple theoretical approximation for the free energy based on the sum of the Madelung energy (energy term) and the

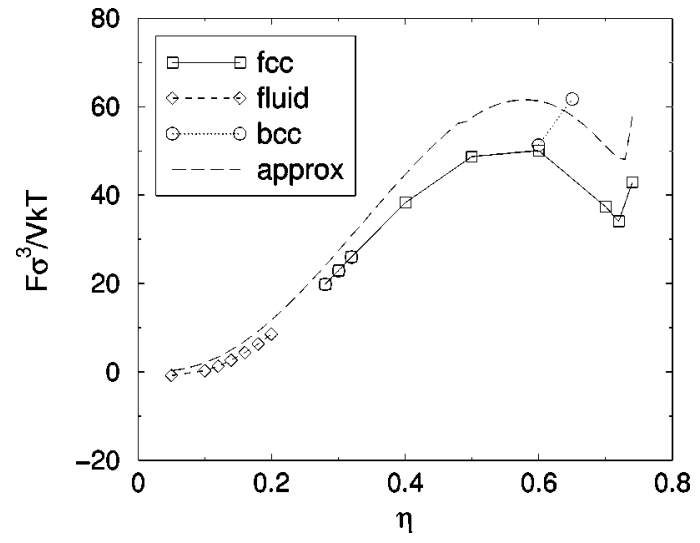

FIG. 3. Free energies per volume at $\kappa \sigma=2.2$ for the fluid, bcc, and fcc phases, resulting from the thermodynamic integration described in Sec. II. Also shown is the result from the approximate theory of Eq. (14).

free energy of a hard-sphere system (entropy term) as

$$
F_{\text {approx }}=N u_{M}+\left\{\begin{array}{cc}
F_{\mathrm{HS}}^{\text {fluid }}, & \eta \leqslant 0.49, \\
F_{\mathrm{HS}}^{\text {sol }}, & \eta>0.49 .
\end{array}\right.
$$

Here the Madelung energy per particle, $u_{M}$, is that of an "infinite" fcc solid and the free energy of the hard-sphere solid, $F_{\mathrm{sol}}^{\mathrm{HS}}$, is obtained from the equation of state by Hall [40]. In Fig. 3, $F_{\text {approx }}$ is plotted with the long-dashed line and, as can be seen, the result is qualitatively the same as the one from the elaborate free energy calculations. This result explains the very broad fluid-fcc coexistence: because of the three-body attraction, at low $\kappa \sigma$ and high densities $(\eta>0.55$ for $\kappa \sigma=2.2)$ the Madelung energy per particle, $u_{M}$, is a monotonically decreasing function of the density. This produces a lowering of the free energy at high densities (in Fig. 3 this is seen at $\eta \approx 0.6$ ). At very high densities $(\eta \approx 0.72$ in Fig. 3) the free energy shoots up again, because there the hard-sphere solid free energy $F_{\mathrm{HS}}^{\text {sol }}$ starts to increase rapidly. Thus the competition between the Madelung energy and the hard-sphere free energy produces a minimum in the fcc free energy curve at high density and explains the broad fluid-fcc coexistence.

In the broad fluid-solid coexistence regime, the fcc crystal is more favorable than the bcc phase as the former phase has a higher maximum packing. The denser packing yields a larger negative three-body energy contribution, which lowers the total free energy of the system. Figure 3 illustrates this: The bcc free energy starts to increase strongly after $\eta \approx 0.6$ due to the hard-core repulsion, while at this packing fraction, the fcc has sufficient space to densify and to lower its free energy due to the three-body attractions.

As the range of the three-body attraction is reduced at higher values of $\kappa \sigma$ (higher salt concentrations), the minimum becomes less pronounced while at the same time the coexisting fluid phase moves to higher densities. Finally, at $\kappa \sigma \approx 2.36$ the fluid is metastable with respect to the bcc, and the fluid-fcc coexistence is replaced by a broad bcc-fcc coexistence. 


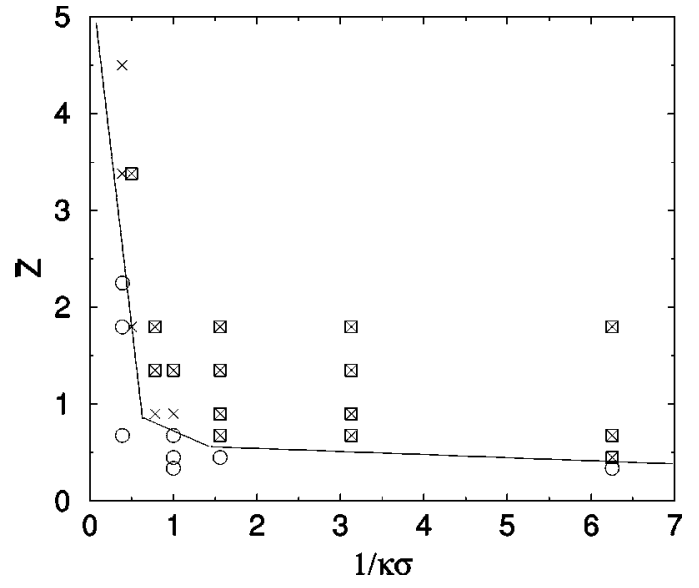

FIG. 4. Crosses $(\times)$ denote the points $(\bar{Z}, \kappa \sigma)$ where free energy calculations based on Eq. (14) predict a gas-solid coexistence, squares $(\square)$ show the points where the van der Waals theory of Ref. [26] predicts a gas-liquid coexistence, and circles $(O)$ show the points where a "narrow" fluid-solid coexistence is predicted by our approach and Ref. [26]. The line is our prediction for the crossover from a "broad" gas-solid to a "narrow" fluid-solid coexistence.

The approximation for the free energy given in Eq. (14) can be used as a quick tool for mapping out the fluid-fcc and bcc-fcc coexistence points in the low- $\kappa \sigma$ regime. ${ }^{1}$ In Fig. 2 the coexistence points obtained from the free energy curves provided by Eq. (14) are denoted by crosses $(\times)$. As can be seen, the results obtained from the approximate method are in good agreement with those from free energy calculations.

We also used Eq. (14) to check the stability of the gasliquid critical point that was predicted for the present system in Ref. [26], on the basis of a simple van der Waals-like theory. For $\bar{Z}=1.8$ the analysis of Ref. [26] yields a gasliquid critical point at $\kappa \sigma \simeq 1.8$ and $\eta \simeq 0.4$; i.e., for $\kappa \sigma<1.8$ there is predicted to be an $\eta$ regime where gas and liquid coexist. Comparison with the present phase diagram for $\bar{Z}$ $=1.8$ in Fig. 2 reveals that this predicted critical point is in fact metastable with respect to fluid-fcc coexistence. The same conclusion holds for the critical points that were identified for other values of $\bar{Z}$ : application of Eq. (14) reveals that a broad fluid-fcc coexistence is thermodynamically more favorable than the gas-liquid transition for all values of $\bar{Z}$ considered. However, the analysis of Ref. [26] is still of use, since the curve in the $(\bar{Z}, \kappa \sigma)$ plane that separated the supercritical from the subcritical regime turns out to be remarkably close to the line that separates a "narrow" fluid-solid regime from a "broad" gas-solid regime (for $\bar{Z}=1.8$ this would take place at $\kappa \sigma \simeq 2.4$ ). This is illustrated in Fig. 4 , where the crosses denote a "broad" gas-solid coexistence

\footnotetext{
${ }^{1}$ The approximate free energy $F_{\text {approx }}$ in Eq. (14) only accounts for the "crude" features of the phase diagram-i.e., either for the broad fluid-fcc or for the broad bcc-fcc phase coexistences. It is unable to predict the phase behavior in the $\kappa \sigma$ regime where these effects are not seen.
}

based on Eq. (14) and the squares denote gas-liquid coexistence as predicted in Ref. [26]. The circles denote a "narrow" fluid-solid regime and the line represents our prediction for the crossover from a "narrow" fluid-solid to a "broad" gassolid regime.

\section{CONCLUSIONS AND DISCUSSION}

We performed a computer simulation study of the phase diagram of a suspension of charged colloids at low salinity, taking into account effective pair and triplet interactions between the colloids. The effective potentials are taken from an earlier Poisson-Boltzmann study [26], and are such that the pair potential is a DLVO-like screened-Coulomb repulsion [see Eq. (3)], whereas the triplet potential is purely attractive [see Eq. (4)].

The main effect of the inclusion of triplet interactions is a reduction of the stability of the bcc phase in favor of the fluid and fcc phase or coexistence of the latter two. The triplet attractions induce, at sufficiently low salinity, a very broad coexistence regime of a dilute fluid with an extremely dense fcc phase, while at intermediate salinity a broad bcc-fcc coexistence regime appears.

There are two reasons for the destabilization of the bcc phase: First, the bcc phase is stabilized by long-range repulsions and, therefore, any attraction (or other disturbances like truncation of the potential, see Ref. [41]) will reduce its stability. Second, in the broad fluid-solid coexistence regime, the three-body attraction favors the fcc solid as it has a higher maximum packing than the bcc phase. The denser packing yields a larger negative three-body energy contribution, which lowers the free energy of the system. The dip in the free energy (see Fig. 3) is beyond the maximum packing $(\eta=0.698)$ of the bcc crystal.

We also conclude that any gas-liquid coexistence that may occur due to the attractive triplet interactions is metastable with respect to gas-fcc coexistence. This is in line with the results of Ref. [42], where it was shown that many-body attractions have a strong tendency to give rise to gas-solid rather than gas-liquid coexistence.

We want to stress the fact that none of the experimentally observed broad coexistence regions can be explained with the pairwise Yukawa picture and that this broad coexistence is always seen in the low-salt regime. Therefore, we suggest that many-body interactions between the colloids, which become important in the low-salt regime, are the reason for these broad coexistence regions.

Finally, some critical remarks on our results must be made, since they are, at least in the low-salt regime, in disagreement with experimental observations: we are not aware of reports of observations of almost-close-packed fcc phases with a dilute gas phase. This suggests that a fully realistic description of charged colloids in this regime requires the inclusion of four-body and higher-order terms as well, which constitutes a daunting task: not only is the calculation of the $n$-body potential $\Omega^{(n)}$ for $n \geqslant 4$ computationally demanding, but including them into an (efficient) Monte Carlo scheme is also far from trivial. However, at high salinity the system is well described by pairwise additivity, and therefore we ex- 
pect that in an intermediate regime of the salt concentration (or $\kappa \sigma$ ) and $\eta$, the system is well described by pair and triplet interactions alone. Where exactly this regime is needs further investigation.

\section{ACKNOWLEDGMENTS}

It is pleasure to thank D. Aarts and H. Lekkerkerker for making us aware of Ref. [4]. This work is part of the re- search program of the "Stichting voor Fundamenteel Onderzoek der Materie" (FOM), which is financially supported by the "Nederlandse Organisatie voor Wetenschappelijk Onderzoek" (NWO). We thank the Dutch National Computer Facilities Foundation for access to the SGI Origin 3800 . The High Performance Computing Group of Utrecht University is gratefully acknowledged for ample computer time. A.-P.H. gratefully acknowledges the financial support from the Finnish Cultural Foundation.
[1] M. Dijkstra, Curr. Opin. Colloid Interface Sci. 6, 372 (2001).

[2] B. Derjaguin and L. Landau, Acta Physicochim. URSS 14, 633 (1941); E. J. W. Verwey and J. Th. G. Overbeek, Theory of the Stability of Lyotropic Colloids (Elsevier, Amsterdam, 1948).

[3] J. M. Victor and J.-P. Hansen, J. Chem. Soc., Faraday Trans. 1 81, 43 (1985).

[4] S. Hachisu, Croat. Chem. Acta 71, 975 (1998).

[5] W. B. Russel, D. A. Saville, and W. R. Schowalter, Colloidal Dispersions (Cambridge University Press, Cambridge, England, 1989).

[6] N. Ise, T. Okubo, M. Sugimura, K. Ito, and H. J. Nolte, J. Chem. Phys. 78, 536 (1983); N. Ise and M. V. Smalley, Phys. Rev. B 50, 16722 (1994).

[7] K. Ito, H. Yoshida, and N. Ise, Science 263, 66 (1994); B. V. R. Tata, E. Yamahara, P. V. Rajamani, and N. Ise, Phys. Rev. Lett. 78, 2660 (1997).

[8] C. P. Royall, M. E. Leunissen, and A. van Blaaderen, J. Phys.: Condens. Matter 15, 3581 (2003).

[9] A. E. Larsen and D. G. Grier, Nature (London) 385, 230 (1997).

[10] B. V. R. Tata, M. Rajalakshmi, and A. K. Arora, Phys. Rev. Lett. 69, 3778 (1992).

[11] T. Palberg and M. Würth, Phys. Rev. Lett. 72, 786 (1994).

[12] L. Belloni, J. Phys.: Condens. Matter 12, R549 (2000); J.-P. Hansen and H. Löwen, Annu. Rev. Phys. Chem. 51, 209 (2000).

[13] Y. Monovoukas and A. P. Gast, J. Colloid Interface Sci. 128, 533 (1989).

[14] E. B. Sirota, H. D. Ou-Yang, S. K. Sinha, P. M. Chaikin, J. D. Axe, and Y. Fujii, Phys. Rev. Lett. 62, 1524 (1989).

[15] H. J. Schöpe, T. Decker, and T. Palberg, J. Chem. Phys. 109, 10068 (1998).

[16] E. L. Pollock and J.-P. Hansen, Phys. Rev. A 8, 3110 (1973).

[17] A.-P. Hynninen and M. Dijkstra, Phys. Rev. E 68, 021407 (2003).

[18] E. J. Meijer and F. El Azhar, J. Chem. Phys. 106, 4678 (1997); F. El Azhar, M. Baus, J.-P. Ryckaert, and E. J. Meijer, ibid. 112, 5121 (2000).

[19] B. Beresford-Smith, D. Y. C. Chan, and D. J. Mitchell, J. Colloid Interface Sci. 105, 215 (1985); D. Y. C. Chan, Phys. Rev. E 63, 061806 (2001).

[20] R. van Roij and J.-P. Hansen, Phys. Rev. Lett. 79, 3082 (1997); R. van Roij, M. Dijkstra, and J.-P. Hansen, Phys. Rev.
E 59, 2010 (1999); R. van Roij and R. Evans, J. Phys.: Condens. Matter 11, 10047 (1999); R. van Roij, ibid. 12, A263 (2000).

[21] P. B. Warren, J. Chem. Phys. 112, 4683 (2000).

[22] M. J. Grimson and M. Silbert, Mol. Phys. 74, 397 (1991).

[23] H. Graf and H. Löwen, Phys. Rev. E 57, 5744 (1998).

[24] M. Brunner, C. Bechinger, W. Strepp, V. Lobaskin, and H. H. von Grünberg, Europhys. Lett. 58, 926 (2002); R. Klein, H. H. von Grünberg, C. Bechinger, M. Brunner, and V. Lobaskin, J. Phys.: Condens. Matter 14, 7631 (2002).

[25] M. Brunner, J. Dobnikar, H. H. von Grünberg, and C. Bechinger, Phys. Rev. Lett. 92, 078301 (2003).

[26] C. Russ, H. H. von Grünberg, M. Dijkstra, and R. van Roij, Phys. Rev. E 66, 011402 (2002).

[27] J. Dobnikar, R. Rzehak, and H. H. von Grünberg, Europhys. Lett. 61, 695 (2003).

[28] A.-P. Hynninen, M. Dijkstra, and R. van Roij, J. Phys.: Condens. Matter 15, S3549 (2003).

[29] R. Car and M. Parrinello, Phys. Rev. Lett. 55, 2471 (1985).

[30] H. Löwen, P. A. Madden, and J.-P. Hansen, Phys. Rev. Lett. 68, 1081 (1992); H. Löwen, J.-P. Hansen, and P. A. Madden, J. Chem. Phys. 98, 3275 (1993).

[31] M. Fushiki, J. Chem. Phys. 97, 6700 (1992).

[32] M. Dijkstra, R. van Roij, and R. Evans, Phys. Rev. E 59, 5744 (1999).

[33] P. Attard, Phys. Rev. A 45, 5649 (1992).

[34] D. Frenkel and B. Smit, Understanding Molecular Simulations, 2nd ed. (Academic Press, London, 2002).

[35] D. Frenkel and A. J. C. Ladd, J. Chem. Phys. 81, 3188 (1984).

[36] J. M. Polson, E. Trizac, S. Pronk, and D. Frenkel, J. Chem. Phys. 112, 5339 (2000). See also Ref. [34].

[37] N. F. Carnahan and K. E. Starling, J. Chem. Phys. 51, 635 (1969).

[38] M. Aubouy, E. Trizac, and L. Bocquet, J. Phys. A 36, 5835 (2003); See also L. Bocquet, E. Trizac, and M. Aubouy, J. Chem. Phys. 117, 8138 (2002); E. Trizac, L. Bocquet, and M. Aubouy, Phys. Rev. Lett. 89, 248301 (2002).

[39] S. Hamaguchi, R. T. Farouki, and D. H. E. Dubin, Phys. Rev. E 56, 4671 (1997).

[40] K. R. Hall, J. Chem. Phys. 57, 2252 (1972).

[41] A.-P. Hynninen and M. Dijkstra, J. Phys.: Condens. Matter 15, S3557 (2003).

[42] R. P. Sear, Phys. Rev. E 61, 651 (2000). 